\title{
Field Simulation as an Aid to Machine Design: The State of the Art
}

\author{
Jan K. Sykulski \\ School of Electronics and Computer Science, University of Southampton, Southampton SO17 1BJ, United Kingdom
}

\begin{abstract}
The paper provides an overview of the modern field simulation techniques available to assist in the design and performance prediction of electromechanical devices, including electric motors. Commercial software, usually based on finite element or related techniques, is already very advanced and provides a reliable tool for every-day use in the design office. At the same time Computational Electromagnetics is a thriving area of research with emerging new techniques and methods, in particular for multi-physics and optimisation problems.
\end{abstract}

\section{INTRODUCTION}

Designers of electrical machines need to satisfy the customer on a number of criteria and be competitive regarding low first and operating costs, high efficiency and reliability, minimum weight, close tolerances, etc. Moreover, new types of machines are being developed and applied. Thus it becomes increasingly essential to be able to analyse any proposed design in considerable detail, so that a near optimum may be obtained.

Recent advances in Computational Electromagnetics, encouraged by continuing increase of power and speed of computers, make finite elements and related techniques an attractive alternative to well established semi-analytical and empirical design methods, as well as to the still popular 'trial and error' approach. There has been important progress in fundamental formulations providing more solid foundations for numerical field analysis. There are specialised conferences and symposia dedicated to development of methods and simulation techniques for magnetic, electric and electromagnetic fields. The two major bi-annual conferences are COMPUMAG [1] (organised by the International Compumag Society) and CEFC [2] (sponsored by the IEEE Magnetics Society), both reporting on recent advances in theory and software methodology in the context of applications to real engineering problems. Although many devices are considered, with both low frequency and high frequency aspects featuring prominently, traditionally the electrical machines community is strongly represented and design issues a routine topic of discussions. There are several smaller, but more focused, regular meetings like CEM (Computation in Electromagnetics), organised by the Professional Network on Electromagnetics of the IEE (Institution of Electrical Engineers, London) with selected papers published as a special issue of IEE Proceedings [3, 4]; ISEF (International Symposium on Electromagnetic Fields in Electrical Engineering) [5]; EPNC (Symposium on Electromagnetic Phenomena in Nonlinear Circuits) [6] and others. The International Conference on Electrical Machines (ICEM) - one of the main big meetings devoted entirely to electrical machines - has an appreciable proportion of papers reporting on field computation techniques and a section devoted specifically to finite element modelling [7], with a selection of extended articles published in COMPEL journal [8].

The activities of the Computational Electromagnetics community are overseen and coordinated by the International Compumag Society [9], an independent organisation with around 700 members from over 40 countries, which has as its mission the advancement and dissemination of knowledge about the application of computer methods to field problems having significant electric, magnetic or electromagnetic components. The ICS Newsletter [10] regularly publishes review articles on hot topics in electromagnetics, often with direct relevance or application to electrical machines. Another form of networking is offered by the IEE through its Professional Network on Electromagnetics [11].

There are several books and monographs introducing the art of field computation to practicing engineers and designers at various levels, from fundamental [12] to advanced [13 - 15]; some are very specifically relating to electrical power engineering in general [16] or design methods for electrical machines in particular [17]. Books on CAD in magnetics are also available [18]. Overall, there is a vast literature on the subject which covers various aspects of field simulations in the context of design and performance prediction of electrical machines.

\section{The Industrial Perspective}

Computational Electromagnetics (CEM), that is to say, the procedures for approximating electromagnetic fields by means of numerical algorithms, is now a mature subject - and an active research discipline in its own right - practised by a large international community serving science and industry. Computer modelling is used at all stages in the design of electromechanical devices and it is clearly recognised that the use of analytical and experimental methods, followed by expensive and inflexible prototyping, is no longer cost-effective. However, it is perhaps true to say that many managers in industry - the very people who would benefit most from using electromagnetic software as an everyday tool to cut design times and costs - still perceive CEM as a kind of "black magic". Moreover, since government funding available for fundamental work in this field is scarce, the industry increasingly needs to be involved more directly. But benefits need to be demonstrated to managers before they commit resources to support fundamental developments. All this may sound only too familiar to many scientists struggling to secure research funding, but there is a message to the community to be more proactive in promoting CEM as an efficient design tool. 
Closely linked with the industrial requirements are educational needs; these depend strongly on the type of users necessitated by industry to run the CEM based design systems efficiently. It may be argued that three categories of users are usually required:

1. those able to run confidently dedicated electromagnetic software, understand field displays, interpret numerical results and incorporate them into design processes;

2. design experts who understand the language of electromagnetics and are capable of creating computational models using available commercial software;

3. electromagnetic software developers - the ultimate CEM experts producing basic computational tools to be used in design offices.

In the early days researchers tended to regard the creation of software as a cultural extension to their work and there was often a free exchange of programs between developers. It is obvious that this is no longer tenable as real costs are involved and software production is a commercial operation. There is no essential difference between hardware and software in this respect; both require development, maintenance and support.

Electromechanical products permeate modern life and it is taken for granted that the designers have made the best possible use of the electromagnetic fields in the device to provide the best performance at least cost. Unfortunately, the discovery of the best choice of size, shape and power characteristics for the components, even using the best of today's computer simulations, is very time consuming and costly; it is therefore likely to be incomplete. There are significant delays in bringing improved products to market and opportunities for even better products are being missed. However, making the subject more appealing both to managers and to students appears to be the crux.

\section{COMMERCIAL SOFTWARE}

This section is not intended to provide a catalogue of all available software in electromagnetics. Nevertheless, it seems worthwhile to mention that there now exist quite a few commercially available systems offering integrated tools for $\mathrm{CAD}$ in magnetics. A typical commercial package will have most of the following components:

- Pre- and Post-Processor: fully interactive, advanced post-viewing facilities, comprehensive range of supported output devices, automatic and adaptive meshing;

- Statics: magneto- and electrostatic analysis with nonlinear (and often anisotropic and hysteretic) materials, including permanent magnets, special versions for laminated materials;

- Steady-state eddy currents: steady-state ac eddycurrent analysis, including complex permeabilities, approximate non-linear solutions (fundamental harmonic field), background dc fields, voltage-driven problems;

- Transient eddy currents: full transient analysis, nonlinear materials, multiple drives and background dc fields;

- Motional eddy currents: uniform motion induced eddy-current analysis (with constant or varying topology);

- Stress and thermal: mechanical stress using forces, or thermal analysis using ohmic heating, calculated from electromagnetic solutions;

- 2D, 2D axi-symmetric and 3D formulations.
The following is a non-exhaustive list, with relevant web links provided under References, of the most popular software packages already used extensively by designers:

- OPERA, Vector Fields Ltd [19];

- MagNet, Infolytica [20];

- Maxwell, Ansoft [21];

- Emag, ANSYS [22];

- FLUX, CEDRAT Software [23];

- MEGA, Bath University [24];

- Integrated Engineering Software [25].

In addition, there are many in-house systems developed in academic and research institutions, some of which are also commercially available. Finally, there exists software written specifically for designing electrical machines, such as SPEED [26], which can link to some of the general purpose finite element packages listed above.

\section{Pioneering Developments in CEM}

A comprehensive survey of the key developments in CEM and their attribution has recently been published [27]. It appears appropriate to recall here some of the great achievements and milestone developments which have contributed to the art of field computation. In fact many of the ground rules can be traced back to the work of Southwell using finite differences in the 1940's [28]. The Finite Element method (FE) grew out of the structural mechanics community serving the aircraft industry [29], and its development was driven by the needs of the industries involved; it was only much later that the method was studied by mathematicians. An important milestone, as far as electromagnetic field problems are concerned, occurred in 1963 with Winslow [30] reporting on a discretisation scheme based on an irregular grid of plane triangles. He used a generalised finite difference scheme but also introduced a variational principle, both giving the same results. The latter approach can be considered equivalent to the FE method and is consequently the earliest example of this technique in electromagnetics. Silvester and co-workers at McGill University advanced the formulation more generally using unstructured meshes and generic higher order elements. The polynomials introduced by Silvester [31] using simplex coordinates allowed most formulations to be accomplished for a prototypal triangle. Then in 1970, came the first application of the method to rotational electrical machines by Chari and Silvester [32].

In the 1970's the CEM community started to come together by exchanging ideas between researchers in academia, national laboratories and industry. The year 1976 was especially significant as it saw the first Compumag Conference being held in Oxford. Several developments took place leading to significant advances in theory, formulations, numerical techniques and algorithms. The Incomplete Cholesky Conjugate Gradient method (ICCG) was introduced for solving large sparse systems of equations $[33,34]$ in which the operation count goes approximately $n \log n$ and is largely independent of bandwidth; the method still provides the basis for most contemporary codes. Another breakthrough was in the now widespread use of the 'Delaunay meshing', with the original idea dating back to 1934 and successful algorithms implemented more recently in 2D [35] and 3D (using tetrahedral elements) [36] including error analysis. 
Kelvin Transformation was also proposed to model the infinite domain in which the exterior space to a sphere (circle) surrounding the actual model is solved as an interior problem [37, 38]; in this way the 'impossible' boundaries at infinity may easily be taken into account. The introduction of 'Edge Elements' and differential forms was another milestone. Known also as 'Whitney forms' these elements were first introduced to the CEM community by Bossavit [39, 40], followed by important works of Biro et al [41] and Tsibouikis et al [42]. It is also claimed that, relative to the usual vector calculus treatment, differential forms make electromagnetism clearer, simpler, and more intuitive [43, 44]. The complexity of Maxwell's equations is reduced and the relationships can be illustrated by simple diagrams [45]. These diagrams highlight the importance of the constitutive equations which are seen to associate energy density with infinitesimal volumes and therefore energy with complete electromagnetic systems. This gives rise to dual energy formulations. Several contributions have been made in this area, e.g. by Hammond [46]; some lead to a geometrical method known as 'tubes and slices' [47].

Of great interest and importance to designers of electrical machines is modelling of various properties of materials, in particular magnetic hysteresis and anisotropy. Various techniques have been proposed of which the most widely used are those based on scalar or vector Preisach models; the fundamental work in this area has been undertaken by Mayergoyz [48]. A very comprehensive review of past and present modelling techniques may be found in [49]. Moreover, new types of materials have emerged in recent years and require novel formulations. Soft magnetic composites made from powder [50] have had a great impact. The claimed benefits are lower cost and faster production, improved thermal performance, and higher frequency capability. Another exciting new type of material is high temperature superconductors, which offer tremendous potential in terms of reducing the size and increasing efficiency of devices. However, they present a significant modelling challenge because of very high nonlinearity and anisotropic properties [51].

Another challenge is presented when applying FE to systems under dynamic conditions, as some form of moving meshes is required. Various elegant solutions have been proposed, including - amongst others - special air-gap elements to couple analytic solutions for the airgap with a standard FE solution [52], the use of Lagrange multipliers to couple independent FE meshes that are free to rotate [53], overlapping meshes [54] and moving band techniques [55].

Finally, it is worth pointing out that - although finite elements have proven by far the most versatile technique for modelling practical engineering devices and systems other methods have been and continue to be developed, including successful implementations in the area of electrical machines. One should mention the Transmission Line Matrix method (TLM) [56, 57] - although with relevance mainly in high frequency area - and the whole family of formulations based on Finite Integration approach (see for example [58]). Of particular significance may be the Boundary Element Method (BEM) [59] favoured by some as only a mesh on the surfaces is required, making the codes easier to use and efficient. However, non-linearity and skin effect are often an issue so hybrid FE-BEM formulations are proposed [60].

\section{The State Of the ArT}

Significant progress in implementation of new techniques has lead to more efficient, faster, more accurate and numerically stable algorithms. Amongst the advances which have recently made the greatest impact on the CEM community, the following should be mentioned:

- a new Finite Element Difference (FED) method,

- higher order Finite Difference Time Domain (FDTD),

- further developments of the Transmission Line Matrix (TLM) methods,

- the Multiple Multipole Technique (MMT),

- the use of Finite Integration Technique (FIT),

- a Subspace Projection Extrapolation (SPE) scheme,

- formulations in terms of differential geometry,

- the usage of total/reduced magnetic vector potential and electric scalar potential,

- implementation of edge and facet elements,

- improved anisotropy and hysteresis models,

- efficient application of Continuum Design Sensitivity Analysis (CDSA),

- multi-objective optimisation.

The already cited conferences COMPUMAG [1], CEFC [2] and others [3 - 9] are a continuing source of information about most recent advances. As an example, two particular areas of development will be elaborated, with which the author has been closely involved, namely the computation of electromagnetic forces and application and modelling of superconducting materials.

Knowledge of total forces and their distribution is one of the most important pieces of information required in the design of electrical machines. The most common methods for force prediction are based on either the Maxwell Stress Tensor (MST) or the Virtual Work Principle (VWP). MST is derived from the Lorentz force expression, whereas VWP relates forces to the change in stored energy. For a comprehensive treatment of the principles behind force formulations, and their implications, the reader is refereed to [61]. The major advantage in using MST is that only a single solution is required; unfortunately there are significant implementation problems when applied to practical numerical solutions (e.g. the need for a very fine mesh in the air-gap region). The VWP, on the other hand, computes forces by a virtual displacement of a body and the associated change in the co-energy of the system. However, the required gradient of the co-energy function is rarely available explicitly and thus at least two field solutions are needed, or more for better accuracy. Many researchers have addressed the problem of how to improve the accuracy and reduce the computational effort, and the reader is referred to the works of Coulomb [62], McFee [63] and Hameyer [64]. The most recent attempt is also worth highlighting of a force computation algorithm based on continuum design sensitivity analysis [65]. The formulation allows the computation of the sensitivity of any global quantity to a perturbation in a parameter to be computed without reference to the underlying numerical computation scheme. In effect, it allows a Virtual Work calculation to be performed without the need for a physical displacement. The resultant expressions are similar to the MST but have the important advantage of the integration taking place on the surface of material rather than in the air outside. The approach can generate global forces as well as force distributions over the surface 
of a body, including the case of zero air gap. Moreover, the force expressions clearly indicate the contributions to the global force from each source of magnetic field. The implementation is simple, independent of the numerical analysis approach taken and can be easily used in combination with commercial software.

Discovery and development of new materials present a modelling challenge and often lead to reformulation of fundamental equations or design methods. We will focus here on recent advances in superconductivity, in particular due to their potential impact on electrical machines industry. Ceramic superconductors were discovered in 1986 and their main advantage is that they can operate at liquid nitrogen temperature $(78 \mathrm{~K})$ - hence the name High Temperature Superconductors (HTS) - and thus offer relatively cheap and reliable technology. With practical current densities of up to 50 times larger than in conventional copper windings they have great potential in electric power applications (generators, motors, fault current limiters, transformers, flywheels, cables, etc.), as losses are significantly reduced and power output per volume increased. From the design point of view they offer a challenge because of very highly non-linear characteristics and anisotropic properties of materials, and due to unconventional design solutions. The ability to predict and reduce all 'cold' losses is of paramount importance. The behaviour and characteristics of the highly non-linear and anisotropic HTS materials is markedly different to conventional conductors. One of the first devices designed, built and successfully tested was a demonstrator transformer [66]; a particularly satisfying result was the two-fold reduction of losses through the introduction of magnetic flux diverters, which reduce an unwanted component of magnetic field in the coil region. Some more general aspects of the design of large HTS power transformers may be found in [67]. Another completed successful design was of a small synchronous generator [68]; in terms of modelling the important issues were no-load tooth ripple losses due to the distortion of the fundamental flux density wave by the stator slotting, and full-load losses that include the effects of the MMF harmonics of the stator winding. The field penetration into the HTS tape was shown to be accurately simulated using various diffusion models $[69,70]$.

Moreover, other new materials are being introduced leading to improved performance but requiring new computational models and revised design principles. Further progress in CEM methods is continually required and currently undertaken research involves: adaptive meshing and reliable error estimation, efficient handling of non-linearity, hysterisis and anisotropy, incorporation of linear movement and rotation of some parts of the device, combined modelling of fields and circuits (e.g. supplying electronic circuitry), coupled and multi-physics problems and integrated design systems.

\section{Computer Aided Design}

As argued in this article and by many other enthusiasts of the CEM techniques, the computer-aided design (CAD) has come of age in the magentics devices industry. However, difficulties are experienced by new users when introduced to the subject. It is thought that the difficulties arise in two areas: (i) an inadequate understanding of relevant electromagnetic theory and (ii) an inability to appreciate the subtleties of numerical modelling. Thus the value of engineering judgement becomes paramount, to avoid regarding the process of field simulation as 'unquestionably conclusive', almost mechanical one, where insufficient thought may be given to the sound formulation of the problem and to the interpretation of results. To put it trivially, the answer can only be as good as the model adopted. A useful 'check list' of questions (based on [16]) which need to be addressed by users attempting to use CAD systems for machine design may include the following:

- Is a 2D model adequate?

- If so, is it necessary to allow for end effects?

- If $3 \mathrm{D}$ is essential, what simplifications can be made?

- What is the most appropriate potential to use?

- How much of the surroundings need to be modelled?

- Do symmetry and/or periodicity conditions exist?

- What other boundary conditions can be assumed?

- Must induced currents be allowed for?

- If so, what is the highest frequency to be considered?

- Are materials non-linear, anisotropic, hysteretic?

- Are all material characteristics available and accurate?

- Which critical areas require fine discretisation?

- Are variants of the base design to be investigated?

- Can second-order effects be neglected?

- Is supplying circuit necessary in the model?

- What quantities are required from the solution?

Clearly the list could continue almost indefinitely, but it does emphasise the importance and pivotal role of the designer in the process, someone who takes full responsibility for the successful outcome and is much more then an 'operator' for launching the software. However, a well designed CAD system will offer as much 'hassle free' automation as possible to allow the designer to concentrate on the main task at hand rather then worrying about the commands, menus and other details of how to operate the software package. Ideally, a successful design of an electrical machine or any other electromechanical device should be optimised; this presents an additional challenge to software designers, as optimal design often necessitates repetitive usage of finite-element solvers, or other numerically intensive field computation.

A direct way of incorporating field modelling into an optimisation loop is to call the FE package every time a function evaluation is required. Although straightforward in implementation, this on-line approach will normally lead to unacceptable computing times, as for each set of selected design parameters a full field analysis needs to be performed. The number of necessary calls to the FE software escalates as the number of design variables increases; moreover, additional calls are normally required to calculate each gradient of the objective function. Although theoretically this is of no consequence, in the design office environment such an approach becomes impractical. Thus significant effort is currently directed at development of optimisation techniques suitable for such computationally intensive problems $[71,72]$. One method, which has recently attracted significant attention, is called surrogate modelling, a functional relationship between the design variable space and the objective function space constructed based on design vectors which have their objective function values known. A type of surrogate model known as kriging appears to be very useful [73]. 
Design has to be considered in the context of general trends in optimisation methods. The role of multiobjective tasks is increasing as practical designs often involve conflicting requirements. Such problems may be converted into single-objective tasks with a priori application of knowledge or imposition of a decision (e.g. weighting factors), but it is argued that information can easily be lost in the process. Instead the application of Pareto Optimal Front (POF) approximation is advocated, where several solutions are optimal in a 'pareto' sense.

Finally, in engineering practice, it is often the improvement to the design, not necessarily a global optimum, which is of interest. Hence the sensitivity analysis is of great value as computing times are not affected by the number of design variables. The Continuum Design Sensitivity Analysis (CDSA) is particularly to be recommended as standard EM software may be used for extracting gradient information [74, 75].

\section{WHAT THE FUTURE HOLDS}

Looking into a crystal ball to predict the future is hardly appropriate for a scientist or an engineer, but it might be worth re-emphasising that Computational Electromagnetics is a very active area of research, the achievements to date are considerable and the tremendous effort continues. General purpose and specialised software packages offer flexible approach to design and virtual prototyping increasingly becomes a norm rather than an exception. One of the challenges is to 'keep up' with the technology; this may be accomplished by regularly monitoring what is reported at relevant conferences and other events. With this in mind the following is a list (with web links provided in References) of recent and forthcoming meetings where further advances in CEM and their relevance to electrical machines design have or are likely to be discussed: CEFC [76], EPNC [77], EMF [78], ICEM [79], IGTE [80], OIPE [81] and COMPUMAG [82].

\section{VIII.CONCLUSIONS}

This paper is an attempt to review the significant advances in the field of Computational Electromagnetics to demonstrate how numerical field simulation could aid the design of electrical machines and devices. Based mostly on the versatile finite element approach, the available software, including general purpose commercial packages, offer a mature tool for performance prediction, optimisation and general design. Tackling the multiphysics problems and multi-objective optimisation are identified as the biggest current challenges.

\section{REFERENCES}

[1] COMPUMAG 2005 Proceedings, IEEE Transactions on Magnetics, vol. 42, No 4, April 2006, ISSN 00189464

[2] CEFC 2004 Proceedings, IEEE Transactions on Magnetics, vol. 41, No 5, May 2005, ISSN 00189464

[3] Special Issue on Computational Electromagnetics, IEE Proceedings; SMT, vol. 149, No 5, Sept 2002, ISSN 13502344

[4] Special Issue on Computational Electromagnetics, IEE Proceedings; SMT, vol. 151, No 6, Nov 2004, ISSN 13502344

[5] ISEF 2005, International Symposium on Electromagnetic Fields in Electrical Engineering, selected papers, COMPEL, Special Issue, vol. 25, No 3, 2006, ISSN 03321649

[6] EPNC 2004, Symposium on Electromagnetic Phenomena in Nonlinear Circuits, selected papers, COMPEL, Special Issue, vol. 25, No 1, 2006.
[7] $16^{\text {th }}$ International Conference on Electrical Machines, Book of digests, 5 - 8 September 2004, ISBN 8392142810

[8] ICEM 2004, International Conference on Electrical Machines, selected papers, COMPEL, Special Issue, Vol.25, No 2, 2006.

[9] International Compumag Society, http://www.compumag.co.uk/

[10] Sykulski J.K. (editor), International Compumag Society Newsletter, ISSN 1026-0854

[11] Professional Network Electromagnetics, IEE, London, UK, http://www.iee.org/OnComms/pn/electromagnetics/

[12] P. Hammond and J.K.Sykulski, Engineering Electromagnetism, Physical Processes and Computation, Oxford Science Publications, New York, 1994.

[13] J.K. Sykulski (editor), Computational Magnetics. Chapman \& Hall, 1995.

[14] R.L. Stoll, The Analysis of Eddy Currents, Clarendon Press, 1974.

[15] K.J. Binns, P.J. Lawrenson and C.W. Trowbridge, The Analytical and Numerical Solution of Electric and Magnetic Fields, John Wiley \& Sons, 1992.

[16] A.B.J. Reece and T.W. Preston, Finite Element Methods in Electrical Power Engineering, Oxford Science Publications, 2000.

[17] K. Hameyer and R. Belmans, Numerical Modelling and Design of Electrical Machines and Devices, WIT Press, 1999.

[18] D.A. Lowther and P.P. Silvester, Computer-Aided Design in Magnetics, Springer, 1985.

[19] OPERA, Vector Fields Ltd, http://www.vectorfields.co.uk/

[20] MagNet, Infolytica, http://www.infolytica.com/en/

[21] Maxwell, Ansoft, http://www.vectorfields.co.uk/

[22] Emag, ANSYS, http://www.ansys.com/

[23] FLUX, CEDRAT Software, http://www.cedrat.com/

[24] MEGA, Bath, http://www.bath.ac.uk/elec-eng/aerc/mega.html

[25] Integrated Engineering Software, http://www.integratedsoft.com/

[26] SPEED, http://www.speedlab.co.uk/software.html

[27] C.W. Trowbridge and J.K. Sykulski, "Some Key Developments in Computational Electromagnetics and their Attribution," IEEE Transactions on Magnetics, vol. 42, No 4, 2006, pp. 503-508.

[28] R. Southwell, Relaxation Methods in Theoretical Physics, OUP, 1946

[29] M.J. Turner et al, "Stiffness and deflection analysis of complex structures," J. Aero Sci., vol. 23, p. 805, 1956

[30] A.M. Winslow, "Numerical Calculation of Static Magnetic Fields in an irregular triangle mesh," University of California Report, UCRL-7784, 1964, J Comput Phys 1, p. 149, 1966

[31] P.P. Silvester, "High-order polynomial triangular finite elements for potential problems," Int. J. Engineering Science, 7, 849-861, 1969

[32] M.V.K. Chari, and P.P. Silvester, "Finite Element Analysis of magnetically saturated dc machines," IEEE Trans. PAS, vol. 89, No 7, 1642-51, 1970 \& vol. 90,No 2, pp. 454-464, 1971

[33] J.A. Meijerink, and V. der Vorst, “An Iterative solution method for systems of which the coefficient matrix is a symmetric $M$ matrix," Maths. Comp., vol. 31, p. 148, 1977.

[34] J. Simkin, and C.W. Trowbridge, "On the Use of the Total Scalar Potential in the Numerical Solution of Field Problems in Electromagnetics," IJNME, vol. 14, p.432, 1978.

[35] Z. Cendes et al, "Magnetic field computation using Delaunay triangulation and complementary finite element methods," IEEE Trans. on Magnetics, vol. 19, 1983.

[36] L. Janucke, and A. Kost, "Error Estimation and Adaptive Mesh Generation in the 2D and 3D Finite Element Method," IEEE Trans. Magn., vol. 32, no. 3, 1992.

[37] E.M. Freeman and D.A. Lowther, "A Novel Mapping Technique for Open Boundary Finite Element Solutions to Poissons Equation," IEEE Trans. Magn., vol. 24, no. 6, Nov 1988.

[38] J. Imhoff, G. Meunier, and J.C. Sabonnadiere, "Finite Element Modelling of Open Boundary Problems," IEEE Trans. Magn., vol. 26, no. 2, 1990.

[39] A. Bossavit, and J.C. Verite, "A mixed FEM-BIEM method to solve 3-D eddy current problem," IEEE Trans. Magn., pp. 431435,1982 . 
[40] A. Bossavit, "Whitney forms: a class of finite elements for threedimensional computations in electromagnetism," IEE Proc. A, vol. 135, pp. 493-500, 1988.

[41] O. Biro, K. Preis, and K. Richter, "On the use of the Magnetic Vector Potential in the Nodal and Edge Finite Element Analysis of 3D Magnetostatic problems," IEEE Trans. Magn., vol. 32, no. 3, 1996.

[42] T. Yioultsis, and T. Tsiboukis, "Multiparametric Finite Elements: a Systematic Approach to the construction of 3-D, Higher Order, Tangential Vector Shape Functions," IEEE Trans. Magn., vol. 32, no. 3, 1996.

[43] D. Baldomir, "Differential forms and electromagnetism in 3dimensional Euclidian space ${ }^{3}$, , IEE Proc A, vol. 133, p. 139, 1986.

[44] Z. Ren, "Application of Differential Forms in the finite element formulation of Electromagnetic Problems," ICS Newsletter, ISSN 1026-0854, vol. 7, no .3, pp. 6-11, 2000.

[45] E. Tonti, "Finite Formulation of Electromagnetic Field," ICS Newsletter, ISSN 1026-0854, vol. 8, no. 1, pp. 5-11, 2001.

[46] P. Hammond, and J. Penman, "Calculation of inductance and capacitance by means of dual energy principles, " Proc IEE, vol. 123, no. 6, pp. 554-559, 1976.

[47] J.K. Sykulski, "Computer package for calculating electric and magnetic fields exploiting dual energy bounds," IEE Proceedings $A$, vol. 135 , no. 3, pp. 145-150, 1988.

[48] I. M. Mayergoyz, "Mathematical models of hysteresis," IEEE Trans. Magn., vol. 22, 1986.

[49] L. Dupre, and J. Malkebeek, "Electromagnetic hysteresis modelling: from material science to finite element analysis of devices," ICS Newsletter, ISSN 1026-0854, vol. 10, no. 3, pp. 4$14,2003$.

[50] A.G. Jack, B.C. Mecrow, P.G. Dickinson, and D. Stephenson, "Permanent-magnet machines with powdered iron cores and prepressed windings," IEEE Trans. on Ind. Appl., vol. 36, pp. 1077-1084, 2000.

[51] J.K. Sykulski, K.F. Goddard, and R.L. Stoll, "A method of estimating the total AC loss in a high-temperature superconducting transformer winding," IEEE Trans. Magn., vol. 36, pp.1183-7, 2000.

[52] A. Razek, J. Coulomb, M. Feliachi, and J.C. Sabonnadiere, "Conception of an Air-Gap Element for the Dynamic Analysis of the Electromagnetic Field in Electric machines," IEEE Trans. Magn., vol. 18, no. 2, 1982.

[53] D. Rodger, H Lai, and P Leonard, "Coupled Elements for Problems Involving Motion," IEEE Trans. Magn., vol. 26, no. 2, 1990.

[54] I.A. Tsukerman, "Overlapping finite elements for problems with movement," IEEE Trans. Magn., vol. 28, No. 5, pp. 2247-2249, 1992.

[55] A. Demenko, "Movement simulation in finite element analysis of electric machine dynamics," IEEE Trans. Magn., vol. 32, no. 3, 1996.

[56] C. Christopoulos, The Transmission-Line Modeling Method: TLM, IEEE Press and Oxford University Press, 1995.

[57] P. Sewell, J.G. Wykes, T.M. Benson, D.W.P. Thomas, A. Vukovic, and C. Christopoulos, "Transmission line modelling using unstructured meshes," IEE Proc SMT, vol. 151, no. 6, pp. 445-448, 2004

[58] T. Weiland, “Time Domain Electromagnetic Field Computation with Finite Difference Methods," Int. Journal of Numerical Modelling, vol. 9, pp. 295-319, 1996.

[59] C.A. Brebbia and A. Kassab, Electrical Engineering and Electromagnetics VII (ELECTROCOMP 2005), Wit Press, 2006, ISBN: 1845640063

[60] Y. Takahashi and S. Wakao, "Large-Scale Analysis of EddyCurrent Problems by the Hybrid Finite Element-Boundary Element Method Combined With the Fast Multipole Method", IEEE Trans. Magn., vol. 42, no. 4, pp. 671-674, 2006.

[61] C.J. Carpenter, "Surface-integral methods of calculating forces on magnetized iron parts," IEE monograph no. 342, pp. 19-28, 1959.
[62] J.L. Coulomb, and G. Meunier, "Finite Element Implementation of virtual work principle for magnetic force and torque computation," IEEE Trans. Magn., vol. 20, 1985.

[63] S. McFee, J. Webb, and D.A. Lowther, "A tunable volume integration formulation for force calculation in finite-element based computational magnetostatics," IEEE Trans. Magn., vol. 24, pp. 439-442, Jan 1988.

[64] F. Henrotte, H.V. Sande, G. Deliege, and K. Hameyer, "Electromagnetic force density in a ferromagnetic material," IEEE Trans. Magn., vol. 40, pp. 553-556, 2004.

[65] D. H. Kim, David A. Lowther, and J. K. Sykulski, "Efficient Force Calculations Based on Continuum Sensitivity Analysis," IEEE Trans. Magn., vol. 41, no. 5, pp. 1404-7, 2005.

[66] J.K. Sykulski, K. Goddard and R.L. Stoll, "High temperature super-conducting demonstrator transformer: design considerations and first test results," IEEE Trans. on Magnetics, vol. 35, no. 5, pp. 3559-61, 1999.

[67] J.K. Sykulski, C. Beduz, R.L. Stoll, M.R. Harris, K. Goddard and Y. Yang, "High temperature superconducting power transformers: conclusions from a design study," IEE Proceedings; Electrical. Power Applications, vol. 146, no 1, pp. 41-52, 1999.

[68] M.K. Mosawi, C.Beduz, K.F. Goddard, J.K. Sykulski, Y. Yang, B. $\mathrm{Xu}$, K.S. Ship, R. Stoll and N.G. Stephen, "Design of a $100 \mathrm{kVA}$ high temperature superconducting demonstration synchronous generator," Physica C, 372-6(P3), pp. 1539-1542, 2002.

[69] K.F. Goddard, J.K. Sykulski and R.L. Stoll, "A new approach to modelling dominant AC loss in HTc superconducting solenoidal windings," IEEE Trans. on Magn.s, vol. 35, no. 3, pp. 1195-8, 1999.

[70] J.K. Sykulski, M. Rotaru and R.L. Stoll, "2D modelling of field diffusion and AC losses in high temperature superconducting tapes," IEEE Trans. on Magn., Vol. 36, no. 4, pp. 1178-82, 2000.

[71] A. Sobester, S.J. Leary and A.J. Keane, "On the design of optimization strategies based on global response surface approximation Models," Journal of Global Optimization, 33, pp. 31-59, 2005.

[72] M. Farina and J.K. Sykulski, "Comparitive study of evolution strategies combined with approximation techniques for practical electromagnetic optimization problems," IEEE Trans. on Magnetics, vol. 37, no. 5, pp. 3216-3220, 2001.

[73] L. Lebensztajn, C.A.R. Marretto, M.C. Costa and J-L. Coulomb, "Kriging: a useful tool for electromagnetic devices optimization," IEEE Trans. on Magnetics, vol. 40, no. 2, pp. 1196-1199, 2004.

[74] D.H. Kim, K.F. Ship and J.K. Sykulski, J. K., "Applying Continuum Design Sensitivity Analysis combined with standard EM software to Shape Optimisation in Magnetostatic Problems," IEEE Trans. on Magnetics, vol. 40, no 2, pp. 1156-1159, 2004.

[75] D.H. Kim, J.K. Sykulski and D. Lowther, "A Novel Scheme for Material Updating in Source Distribution Optimization of Magnetic Devices using Sensitivity Analysis," IEEE Trans. on Magnetics, vol. 41, no. 5, pp. 1752-1755, 2005.

[76] Twelfth Biennial IEEE Conference on Electromagnetic Field Computation CEFC 2006, Miami, Florida USA, 30 April - 3 May, 2006, http://www.cefc2006.com/

[77] Electromagnetic Phenomena in Nonlinear Circuits, Maribor, Slovenia, 28 - 30 June 2006, http://ptetis.put.poznan.pl/XIXepnc/

[78] Electric and Magnetic Fields, From Numerical Models to Industrial Applications, EMF 2006, Aussois (France), $19-22$ June 2006, http://www.conf-aim.skynet.be/emf2006/

[79] ICEM, International Conference on Electrical Machines, Chania, Crete, Greece, 2-5 Sept 2006, http://www.ntua.gr/ICEM2006/

[80] IGTE Symposium on Numerical Field Calculation in Electrical Engineering, Graz University of Technology, Austria, $17-20$ September, 2006, http://www.igte.tugraz.at/symp06/index.html

[81] Workshop on Optimization and Inverse Problems in Electromagnetics OIPE, Sorrento (Italy), 13 - 15 September 2006, http://143.225.92.163/?who=workshop

[82] COMPUMAG 2007, Aachen, Germany, 24 - 28 June 2007 http://www.compumag.co.uk/compumag_07.html 